\title{
WHAT SHAPES THE COMMUNITIES OF LEARNERS IN A MEDICAL SCHOOL
}

\author{
M. Saqr, J. Nouri, U. Fors \\ Stockholm University (SWEDEN)
}

\begin{abstract}
A positive association between social ties has been reported between social relationships or peer interactions and better performance. However, these findings were reported using traditional descriptive methods that suffered endogeneity, positing a serious threat to the inferences made. Moreover, little is known about how the networks of friendships in a medical school form and what factors derive the community structure. This study was done to evaluate the factors that shaped the social structure of medical students' communities with particular emphasis on the role of academic performance and gender differences. The results of the correlation test between the social popularity measures and performance were statistically significant in the male group and insignificant in the female group. This variance might point out to a different mechanism of community building and social ties that differs among genders. To investigate the factors that affect community building, we used exponential-family random graph models to model the networks and identify the factors that best predict the emergence of ties. The male network included 69 nodes and 365 edges. Besides reciprocity, triangle closure, the city of residence, out-degree and in-degree popularity; the academic performance was a significant factor in terms of both the GPA and the difference between grades of both nodes. In the female network, (50 nodes and 176 edges), academic performance was a not significant factor, both the GPA and the difference between grades of both nodes; while reciprocity, triangle closure, the city of residence, out-degree and in-degree popularity were. The final model in male and female network showed a high degree of goodness-of-fitness statistics. These results highlight the issue of homophily on performance, as a significant factor in how males in this study build their friendship network in contrast to females. It also emphasizes the need for better inferential models that genuinely capture the network effect on performance before jumping to conclusions using traditional descriptive models that suffer the risk of endogeneity.
\end{abstract}

Keywords: Social network analysis, academic performance, community building, interactions, Statistical network modeling, learning analytics.

\section{INTRODUCTION}

The interactions among students trigger the dissemination of knowledge through the inter-links and ties that help them transfer the information into the learning communities where they belong [1]. These interactions facilitate the distribution of information, enhance the diversity of perspectives and provide access to resources [1-4]. Ties provide more than information; they support the social well-being of learners, act as role models and help students manage stress [5-8]. Connected students and the ties between them form the different types of networks that shape, influence and mediate interactions in a tangible way $[6,9,10]$.

A positive association between social ties or peer interactions and academic achievement have been previously reported [11-13] . Baldwin et al. [13] reported a positive correlation between the number of ties in the advisers network and students' performance in an MBA program, Smith et al. [14] reported a positive association between the number of incoming ties from students asking for information and the academic performance in a large course based on traditional lectures. Hommes et al. [15] studied a large cohort of first-year medical students and reported a positive correlation between academic performance with outgoing ties (giving information) or incoming ties (asking for information).

There are two views of the effects of relationships; on the one hand, some argue that the association between relationships and performance might be because students might select their peers based on similarity or homophily by performance. Thus, well-performing students could favor relationships to similar well-performing students [16]. On the other hand, there is considerable evidence of significant 
peer effects in education, behavior, and wellbeing [3, 14, 17-20]. However, the evidence on peer effects used classical statistical techniques [21, 22]. Classical statistical techniques typically assume independence between the outcome and the variables, these assumptions are not possible using network data, because empirical network data are relational by design, being collected from networked actors who happen to react, interact and influence each other [21, 22]. Special statistical approaches were developed for inferential analysis of empirical network data such as exponential random graph models (ERGM) and permutation tests [23]. For the study of the influence of the network on individuals or of outcomes that results from having relations or occupying a certain position, permutation-based tests are both powerful and credible [24].

This study was done to evaluate the factors that shaped the social structure of medical students' communities with particular emphasis on the role of academic performance and gender differences. The following research questions were formulated:

1. Is there a correlation between academic performance and social relationships?

2. Is academic performance a generative factor behind social relationship?

\section{METHODOLOGY}

This study took place at the medical school of Qassim University. Qassim University consist of students that study the same curriculum and that share similar sociocultural and academic contexts. The data collection was done using a survey distributed to all students in the last medical school year in the second semester. The survey contained an informed consent statement, listing the aim of research, protection of privacy procedure and ethical handling of data. The survey instrument included questions about GPA, age, gender, income, place of residence and list of close friends. A total of 119 students returned the survey. Two non-weighted directed networks were constructed based on the nominations of students. Attributes such as age and residence were later added to the network. Indegree centrality (number of nominations by friends) and outdegree centrality (number of friends) were calculated as measures of popularity. Complete anonymisation of data was then made so that students can never be identified or recognized by the researcher or others.

A Bayesian t-test was performed for comparison of means, Bayesian t-test has a good validity and reliability that may supersedes traditional t-test, particularly when the data contains outliers, such as a very popular student or an isolated one [25]. Permutation based nonparametric Spearman correlation coefficient test was performed to assess the correlation between popularity measures and performance.

To investigate the factors that affect community building in both genders, we used "exponentialfamily random graph models" (ERGMs) implemented in Statnet R package to model the networks and identify the factors that best predict the emergence of ties. ERGMs has emerged as the preferred method for inference in social networks as it is not affected by interdependence and can yield results that can be interpreted as those of regression models [22, 24, 26-28]. The generative models are defined by predictors that might explain the probability of emergence of a tie such as age, or gender. Certain configurations of the ties (terms) are also important to the formation of ties, such as reciprocal friendships ties, which has the term (mutual); nodematch (factor) which adds a factor shared by both nodes such as city of residence or age. Other terms used in the study are nodecov, which adds the effect of a node covariate such as grade; absdiff which represents the absolute difference between the nodes' covariate; Gwesp, a term used for shared partners, and ctriple, a term used for triangle formation. for a full list and deeper explanation of ERGM terms please refer to [29]. We tried different parameters and configurations and compared the resulting models using Akaike information criterion, Markov chain Monte Carlo diagnostics, and goodness-of-fitness. The best model that accurately described our network and showed the best diagnostics was reported in the results 


\section{RESULTS}

The male network included 69 nodes and 365 edges; the female network included 50 nodes and 176 edges. A comparison between the networks of both genders using Bayesian t-test shows that females tended to have smaller group of friends (alters) or out-degree centralities, received fewer nominations (fewer in-degree centralities). The difference was statistically significant, an indication of the tendency of female students to have smaller groups, a finding that is consistent with previous research and reviews that found that females tend to have smaller yet close prosocial friendship groups, while males tend to have larger groups with well-defined hierarchies [30-32].

\begin{tabular}{|c|c|c|c|c|c|c|}
\hline Parameter & Group & Mean & SD & $\begin{array}{l}\text { 95\% Credi } \\
\text { Lower }\end{array}$ & $\begin{array}{l}\text { val } \\
\text { er }\end{array}$ & BF10 \\
\hline \multirow[b]{2}{*}{ indegree } & $\mathrm{F}$ & 3.76 & 1.88 & 3.16 & 4.35 & \multirow[t]{2}{*}{54.865} \\
\hline & $M$ & 5.3 & 2.34 & 4.74 & 5.87 & \\
\hline \multirow{2}{*}{ outdegree } & $\mathrm{F}$ & 4.24 & 1.61 & 3.74 & 4.75 & \multirow[t]{2}{*}{$74.489^{*}$} \\
\hline & $\mathrm{M}$ & 5.3 & 1.35 & 4.98 & 5.63 & \\
\hline
\end{tabular}

$\mathrm{BF}=$ Bayesian factor, ${ }^{*} \mathrm{BF}>$ are statistically significant.

\section{RQ1: Is there a correlation between academic performance and social relationships?}

There was no correlation between outdegree centrality (number of friends) and performance in both gender groups. In the male group, there was a statistically significant correlation between the indegree centrality and academic performance, an indication that popular males were more likely to perform better. However, this might have resulted from the fact that students with high performance tend to be friends or homophily on performance. To investigate this claim, we will look if academic performance was a factor that resulted in the ties (a generative factor). This is done through ERGM. And is the subject of the second research question. 

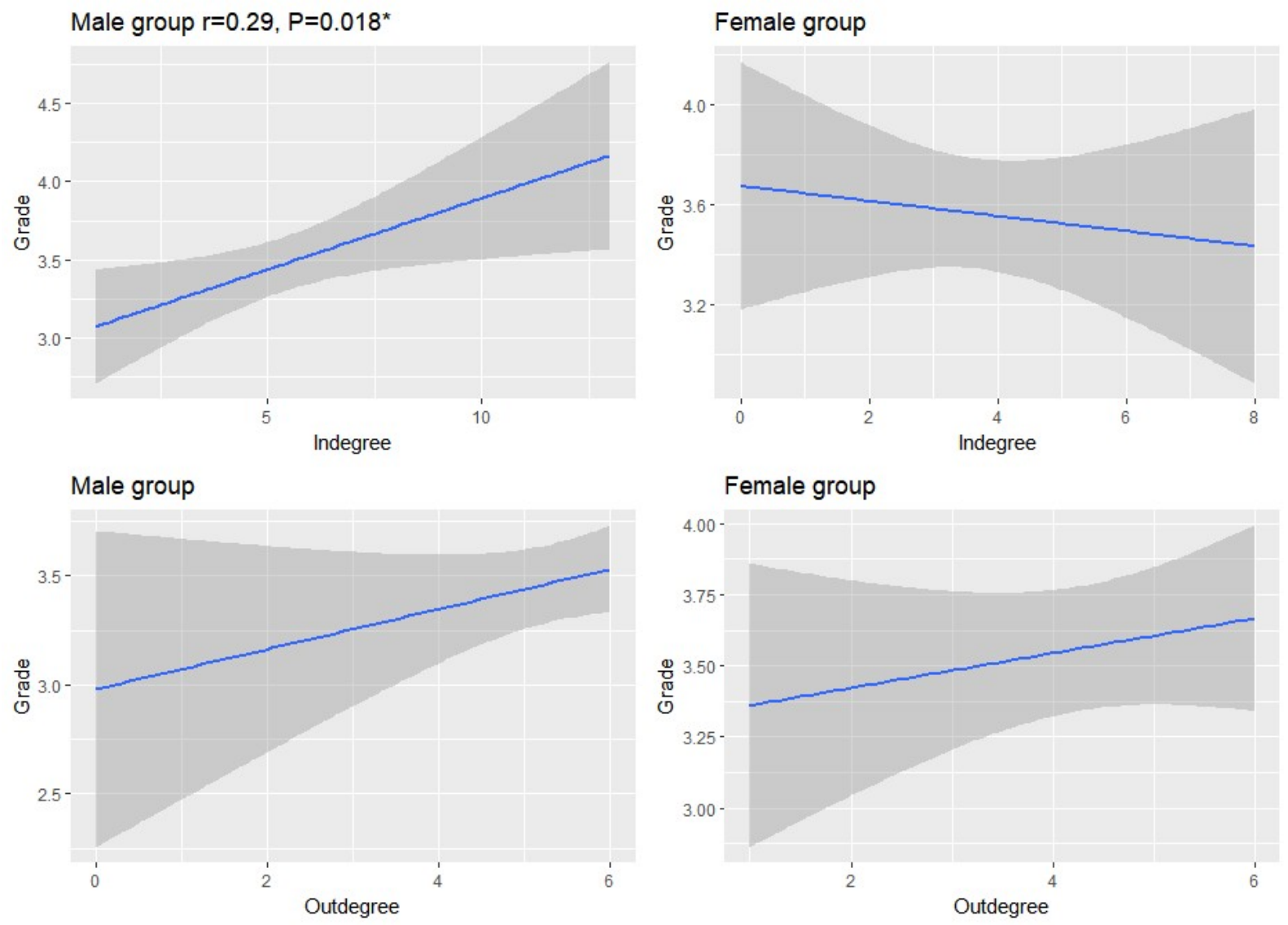

Figure 1 correlation between popularity measures and grades in males and females which was only significant in the male group indegree.

\section{RQ2: Is academic performance a generative factor behind social relationship?}

We used exponential-family random graph models to model the networks and identify the factors that best predict the emergence of ties. The Monte Carlo estimates can be read in a similar way to regression models, where the parameter Edges is treated as the intercept. As shown in table (2), in the male network the significant parameters were reciprocity, triangle closure, the city of residence, out-degree, and in-degree popularity. Besides, the academic performance was a significant factor both the GPA and the absolute difference between grades of both nodes. This is a clear indication that the correlation reported before is probably a result of preferential attachment of high performing students to each other or homophily on performance. In the female network in table (3), the academic performance was a not significant factor, in terms of both the GPA and the difference; while reciprocity, triangle closure, the city of residence, out-degree and in-degree popularity were. This result is consistent with the correlation results that reported no significant correlation. Taken together, we can conclude that the correlation between performance and ties was positive when performance was the reason that the ties emerged, i.e. the ties were the result of performance and not the other way around.

Table 2 Male network parameters

\begin{tabular}{|l|c|c|l|}
\hline Term & Estimate & Std. Error & p-value \\
\hline Edges & 7.69 & 3.09 & $0.01^{*}$ \\
\hline Mutual & 1.90 & 0.22 & $<0.001^{\text {*** }}$ \\
\hline Grade (nodecov) & 0.21 & 0.07 & $0.002^{* *}$ \\
\hline Grade Difference (absdiff) & -0.30 & 0.06 & $<0.001^{* * *}$ \\
\hline Outdegree & -1.57 & 0.26 & $<0.001^{\text {*** }}$ \\
\hline
\end{tabular}




\begin{tabular}{|l|c|l|l|}
\hline Indegree & -0.35 & 0.11 & $0.002^{* *}$ \\
\hline $\begin{array}{l}\text { Shared partners } \\
\text { (GWESP) }\end{array}$ & 1.24 & 0.13 & $<0.001^{* * *}$ \\
\hline Age & -0.11 & 0.05 & $0.02^{*}$ \\
\hline City & 0.44 & 0.08 & $<0.001^{* * *}$ \\
\hline Triangles (ctriple) & 0.44 & 0.11 & $0.001^{* * *}$ \\
\hline
\end{tabular}

Table 2 Female network parameters

\begin{tabular}{|l|r|r|l|}
\hline Term & Estimate & Std. Error & p-value \\
\hline Edges & -1.86 & 4.13 & 0.66 \\
\hline Mutual & 3.46 & 0.42 & $<0.001^{* * *}$ \\
\hline Grade (nodecov) & -0.01 & 0.08 & 0.87 \\
\hline Grade Difference (absdiff) & -0.18 & 0.10 & 0.08 \\
\hline Outdegree & -0.77 & 0.22 & $<0.001^{* * *}$ \\
\hline Indegree & -0.40 & 0.19 & $0.03^{*}$ \\
\hline Shared partners (gwesp) & 1.03 & 0.21 & $<0.001^{* * *}$ \\
\hline Age & 0.01 & 0.07 & 0.87 \\
\hline City & 0.30 & 0.10 & $0.002^{* *}$ \\
\hline Triangles (ctriple) & 0.14 & 0.25 & 0.58 \\
\hline
\end{tabular}

Markov chain Monte Carlo diagnostics and goodness-of-fitness showed that models were accurately describing the networks. In figure (1), we plot the goodness-of-fitness of the whole model, comparing the observed network statistics (thick horizontal line) and the simulated network statistics (boxplots). Since the line is very close to the median line in the boxplot of each network parameter, it means that the model showed excellent goodness-of-fitness and reliably captured the model parameters. Another plot in figure (2) for the goodness-of -fitness of indegree centrality, as it is the most important to our research question. It also shows that the thick line is mostly close to the median line and always in between the upper and lower limits. Which again confirms the reliability of the models. 

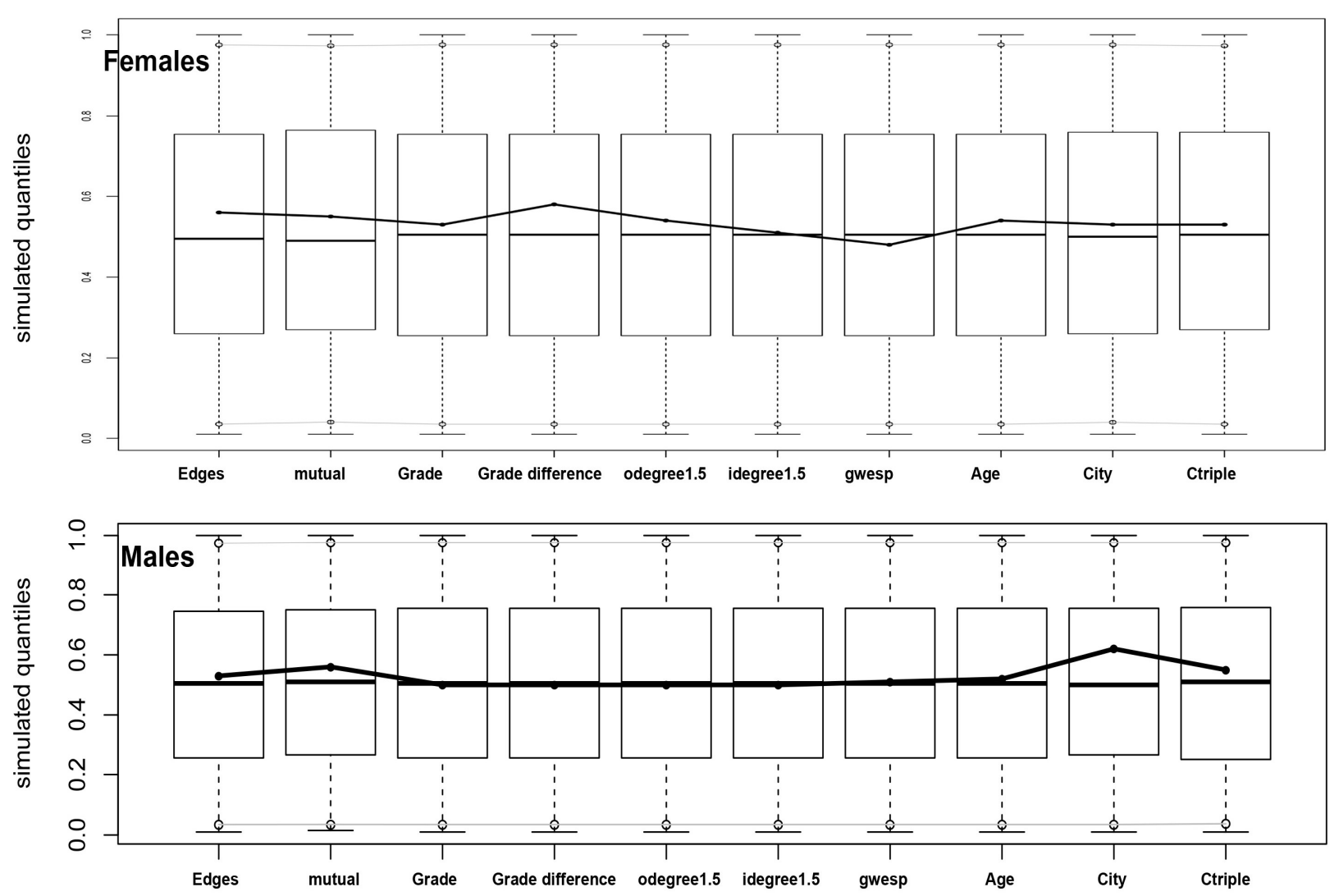

Figure 1 goodness-of-fit ness of all network parameters. Showing the observed statistics (thick horizontal line) very close to the median line in the boxplot of each network parameter.
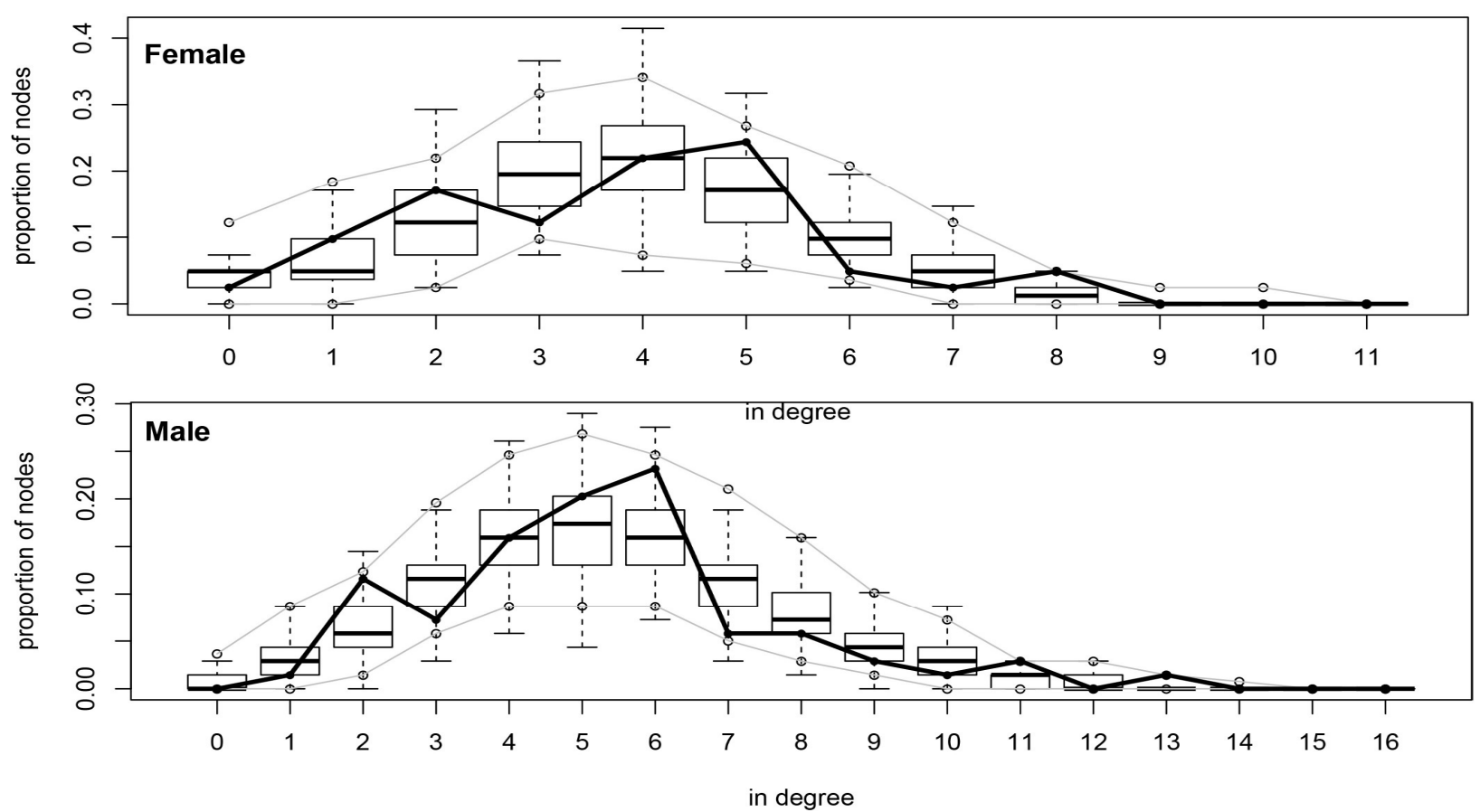

Figure 1 goodness-of-fitness of indegree parameter, showing the observed statistics (thick line) close to the median line in the boxplot of each network parameter. 


\section{DISCUSSION}

A positive association between social ties has been reported between social relationships or peer interactions and better performance. However, these findings were reported using traditional descriptive methods that suffered endogeneity, positing a serious threat to the inferences made. This study was performed to study the correlation between social ties and performance using inferential statistical methods that can be reliably used with empirical network data such as exponential random graph models (ERGM) and permutation tests [21-23].

A long-held belief exists that social ties are a source of social capital. In other words, the wealth of social capital provides resources that help individual achieve their goals and is an important source of support [1-4]. However, it might not apply everywhere and in all environments. In a competitive environment as the medical school where students are top of their classes and their admission to the medical school is based on their scores, the story might be different. Another factor that needs to be considered is the gender factor; the patterns of friendship can be based on different factors among genders.

For instance, our results have shown that the males in this study have larger networks in terms of number of friends. Male networks were based on social factors such as mutual relationships and shared partners, but also on performance. The high performing male students tended to have more friends, and they preferred to attach to similar high performers. As such, correlation with the performance was a result of preferential attachment. It is reasonable here to conclude that preferential attachment to similar high performers is the reason for this positive correlation. Assuming that having more friends has helped students achieve should be taken with caution or probably refuted. In the female group on the other hand, the network was smaller in size, was not based on performance, and there was no correlation with the number of friends and performance. This confirms the fact that when the preferential attachment is not a factor behind the friendship ties, a correlation with the performance was not obtainable.

\section{CONCLUSIONS}

These results highlight the issue of homophily on performance, as a significant factor in how learners build their communities. The high performing male students tended to have more friends, and they preferred to attach to similar high performers. As such, correlation with the performance was a result of preferential attachment. The results also emphasize the need for better inferential models that genuinely capture the network effect on performance before jumping to conclusions using traditional descriptive models that suffer the risk of endogeneity.

\section{ACKNOWLEDGMENTS}

\section{REFERENCES}

1. Siemens G (2014) Connectivism: A Learning Theory for the Digital Age. Int J Instr Technol Distance Learn 1:1-8

2. Burt RS (2002) The social capital of structural holes. New Dir Econ Sociol 201-247

3. Dika SL, Singh K (2002) Applications of Social Capital in Educational Literature: A Critical Synthesis. Rev Educ Res 72:31-60

4. Saqr M, Fors U, Tedre M (2018) How the study of online collaborative learning can guide teachers and predict students' performance in a medical course. BMC Med Educ 18:1-14

5. Borgatti SP, Jones C (1998) Network Measures of Social Capital. Connections 21:27-36

6. Kovanovic V, Joksimovic S, Gašević D, Hatala M (2014) What is the Source of Social Capital ? 
The Association Between Social Network Position and Social Presence in Communities of Inquiry. Proc Work Graph-based Educ Data Min Educ Data Min Conf (G-EDM 2014) 1183:1-8

7. Lin N (1999) Building a Network Theory of Social Capital. Connections 22:28-51

8. Ryan AM, Development C, Development C, August J, Ryan AM (2016) The Peer Group as a Context for the Development of Young Adolescent Motivation and Achievement Published by: Wiley on behalf of the Society for Research in Child Development Stable URL: http://www.jstor.org/stable/1132433 REFERENCES Linked references ar. 72:1135-1150

9. Kop R, Hill A (2008) Connectivism: Learning theory of the future or vestige of the past? Int. Rev. Res. Open Distance Learn. 9:

10. Saqr M, Alghasham Kamal, Habiba. A (2014) The Study of Online Clinical Case Discussions with the Means of Social Network Analysis and Data Mining Techniques. AMEE

11. Saqr M, Fors U, Tedre M, Nouri J (2018) How social network analysis can be used to monitor online collaborative learning and guide an informed intervention. PLoS One 1-22

12. Saqr M, Fors U, Tedre M (2017) How learning analytics can early predict under-achieving students in a blended medical education course. Med Teach 39:757-767

13. Baldwin TT, Bedell MD, Johnson JL (1997) The social fabric of a team-based MBA Program: Network effects on student satisfaction and performance 4. Acad Manag J 40:1369-1397

14. Smith RA, Peterson BL (2007) "Psst ... what do you think?" the relationship between advice prestige, type of advice, and academic performance. Commun Educ 56:278-291

15. Hommes J, Rienties B, de Grave W, Bos G, Schuwirth L, Scherpbier A (2012) Visualising the invisible: A network approach to reveal the informal social side of student learning. Adv Heal Sci Educ 17:743-757

16. Brechwald WA, Prinstein MJ (2011) Beyond homophily: A decade of advances in understanding peer influence processes. J Res Adolesc 21:166-179

17. Sacerdote B (2011) Peer Effects in Education: How Might They Work, How Big Are They and How Much Do We Know Thus Far? In: Handb. Econ. Educ., 1st ed. Elsevier B.V., pp 249-277

18. Griffith AL, Rask KN (2014) Peer effects in higher education: A look at heterogeneous impacts. Econ Educ Rev 39:65-77

19. Gašević D, Zouaq A, Janzen R (2013) “Choose Your Classmates, Your GPA Is at Stake!" The Association of Cross-Class Social Ties and Academic Performance. Am Behav Sci 57:14601479

20. Joksimović S, Manataki A, Gašević D, Dawson S, Kovanović V, de Kereki IF (2016) Translating network position into performance. In: Proc. Sixth Int. Conf. Learn. Anal. Knowl. - LAK'16. ACM Press, New York, New York, USA, pp 314-323

21. Borgatti SP, Everett MG, Jhonson JC (2013) BOOK: Analyzing social networks.

22. Cranmer SJ, Leifeld P, McClurg SD, Rolfe M (2017) Navigating the Range of Statistical Tools for Inferential Network Analysis. Am J Pol Sci 61:237-251

23. Robins $G$, Pattison $P$, Kalish $Y$, Lusher $D$ (2007) An introduction to exponential random graph $\left(\mathrm{p}^{*}\right)$ models for social networks. Soc Networks 29:173-191

24. Newman MEJ, Watts DJ, Strogatz SH (2011) Exponential Random Graph Models for Social Networks. Sage Handb Soc Netw Anal. doi: 10.4135/9781446294413.n32

25. Kruschke JK (2012) Bayesian estimation supersedes the t test. J Exp Psychol Gen 142:573603

26. Hunter DR, Handcock MS, Butts CT, Goodreau SM, Morris M (2008) ergm : A Package to Fit, Simulate and Diagnose Exponential-Family Models for Networks. J Stat Softw. doi: 10.18637/jss.v024.i03

27. Goodreau S, Kitts J, Morris M (2009) Birds of a feather, or friend of a friend? Using exponential random graph models to investigate adolescent social networks. Demography 46:103-125

28. Cranmer SJ, Desmarais BA (2011) Inferential network analysis with exponential random graph 
models. Polit Anal 19:66-86

29. Morris M, Handcock MS, Hunter DR (2008) Specification of Exponential-Family Random Graph Models: Terms and Computational Aspects. J Stat Softw 24:1548-7660

30. Rose AJ, Rudolph KD (2006) A review of sex differences in peer relationship processes: Potential trade-offs for the emotional and behavioral development of girls and boys. Psychol Bull 132:98-131

31. Mehta CM, Strough JN (2009) Sex segregation in friendships and normative contexts across the life span. Dev Rev 29:201-220

32. Koller S, Carlo G, Knight GP, Guzman MRT De, Carlo G, Ontai LL, Koller SH, Knight GP (2004) Gender and Age Differences in Brazilian Children's Friendship Nominations and Peer Sociometric Ratings Gender and Age Differences in Brazilian Children 's Friendship Nominations and Peer Sociometric Ratings. 51: 